Supporting Information for

\title{
Evidence for Facilitated Surface Transport During Ge Crystal Growth by In in Liquid Hg-In Alloys at Room Temperature
}

Dhruba Pattadar, ${ }^{1}$ Yifan Zhao, ${ }^{1}$ Quintin Cheek, ${ }^{1}$ Andrea Sartori, ${ }^{2}$ Rajendra P. Giri, ${ }^{2}$ Bridget Murphy, ${ }^{2,3}$ Olaf Magnussen, ${ }^{2,3 *}$ and Stephen Maldonado ${ }^{1,4^{*}}$

1) Department of Chemistry

University of Michigan

$930 \mathrm{~N}$ University

Ann Arbor, Michigan 48109-1055

2) Institute for experimental and Applied Physics,

Kiel University

Leibnizstr. 11-19,

D-24118 Kiel

Germany

3) Ruprecht Haensel Laboratory

Kiel University

D- 24118 Kiel

Germany

4) Program in Applied Physics

University of Michigan

$930 \mathrm{~N}$ University

Ann Arbor, Michigan 48109-1055

* To whom correspondence should be addressed.

Phone: +1-734-647-4750. e-mail: smald@umich.edu; Home Page: http://www.umich.edu/ mgroup/

Phone: +49-431-880.5579. e-mail: magnussen@physik.uni-kiel.de 


\section{S1. Introduction}

This document contains chronoamperometric responses for $\mathrm{Hg}, \mathrm{Hg}_{1-\mathrm{x}} \mathrm{In}_{\mathrm{x}}$, and In electrodes immersed in $10 \mathrm{mM} \mathrm{Na}_{2} \mathrm{~B}_{4} \mathrm{O}_{7}$ and $50 \mathrm{mM}$ $\mathrm{GeO}_{2}$ solution, a tabulated collection of the fitting parameters used in the first layer model for analysis of the X-ray reflectivity data presented in the main text as well as the corresponding values for two relevant comparison fits for liquid $\mathrm{Hg}$ and liquid In previously reported in the

literature, a tabulated summary of the voltammetric features of the data shown in the main text, a tabulated summary of the Raman spectral features shown in the main text, and a tabulated summary of the X-ray diffraction data presented in the main text. 


\section{S2. Chronoamperometric Responses}
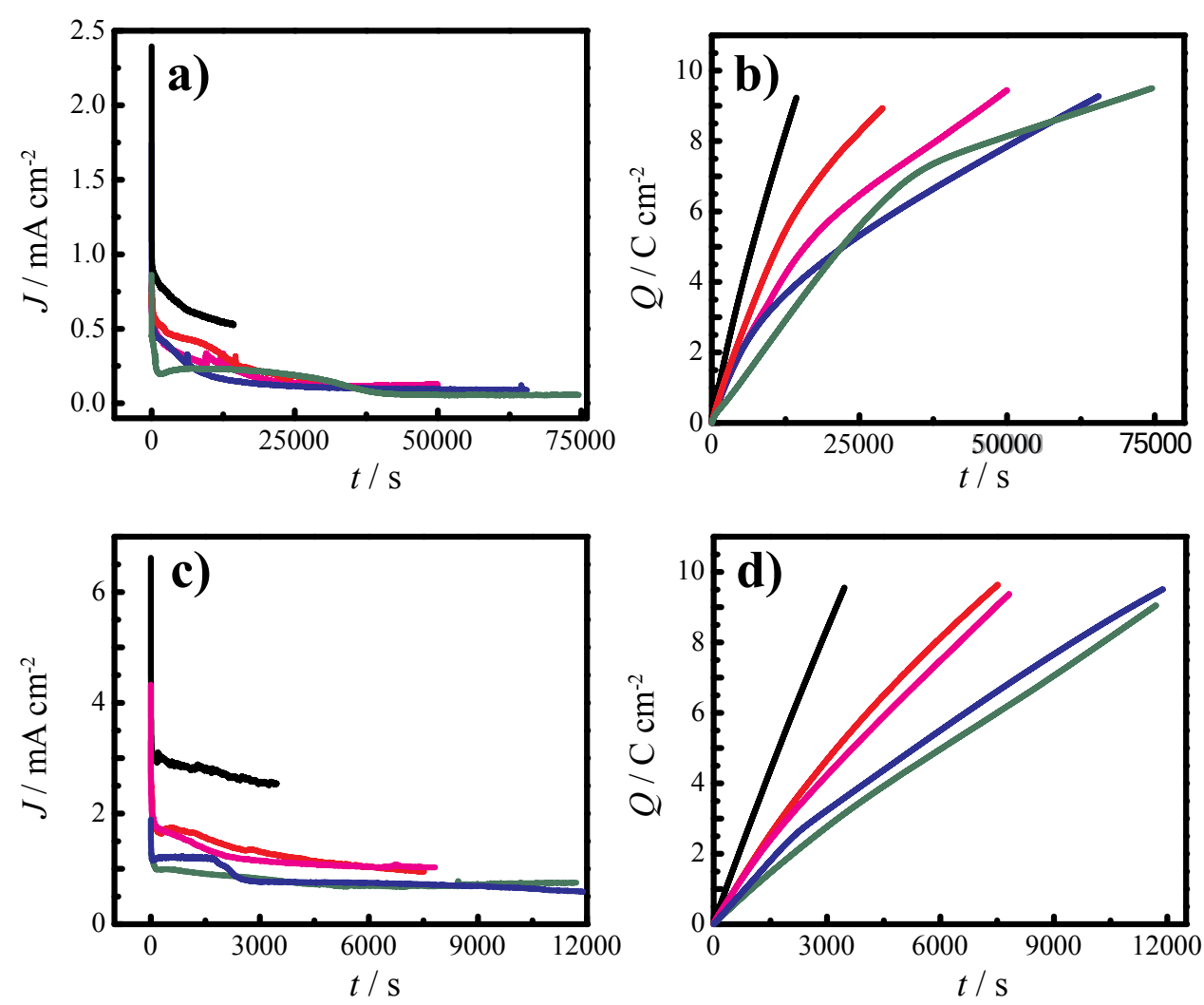

Figure S1. Amperometric responses for $\mathrm{Hg}, \mathrm{Hg}_{1-\mathrm{x}} \mathrm{In}_{\mathrm{x}}$, and In electrodes immersed in $10 \mathrm{mM} \mathrm{Na}_{2} \mathrm{~B}_{4} \mathrm{O}_{7}$ and $50 \mathrm{mM} \mathrm{GeO}_{2}$ solution at a) $-1.4 \mathrm{~V}$ and c) $-1.9 \mathrm{~V}$ vs $E(\mathrm{Ag} / \mathrm{AgCl})$. The corresponding charge passed over time profiles are shown in b) and d), respectively. Green, blue, magenta, red and black lines represents the $\mathrm{Hg}$, $\mathrm{Hg}_{0.7} \mathrm{In}_{0.3}, \mathrm{Hg}_{0.5} \mathrm{In}_{0.5}, \mathrm{Hg}_{0.3} \mathrm{In}_{0.7}$ and In electrodes, respectively. 


\section{S3. Tabulated Data}

The parameters resulting from fits of the normalized XRR data using the first layer modified-distorted crystal model as described in equation 1 in the main paper are tabulated in Table S1. In addition, XRR fit parameters from previously published XRR data for liquid Hg and liquid In are presented for comparison. The data for $\mathrm{Hg}$ immersed in $\mathrm{NaF}(\mathrm{aq})$ are reported for $-0.85 \mathrm{~V}$ at $294 \mathrm{~K}$ are comparable to the experimental measurements reported here within the error margin. The data for liquid In in vacuum at the melting point are presented since bulk liquid In in water at standard pressure is not tenable.

Table S1. Comparis on of X-ray Reflectivity Fitting Parameters for Liquid $\mathbf{H g}, \mathbf{H g}_{0.3} \mathbf{I n}_{0.7}$, and In

\begin{tabular}{|c|c|c|c|c|c|}
\hline $\begin{array}{r}\text { Fitting } \\
\text { Parameter } \\
\end{array}$ & Unit & $\mathrm{Hg}$ in $0.1 \mathrm{M} \mathrm{NaB}_{4} \mathrm{O}_{7}(\mathrm{aq})^{a}$ & $\mathrm{Hg}_{0.3} \mathrm{In}_{0.7}$ in $\left.0.1 \mathrm{M} \mathrm{NaB}_{4} \mathrm{O}_{7(} \mathrm{aq}\right)^{b}$ & $H g$ in $0.1 \mathrm{MNaF}(\mathrm{aq})^{c}$ & $\operatorname{In}^{d}$ \\
\hline$\sigma_{i}$ & $\AA$ & $1.03 \pm 0.01$ & $0.76 \pm 0.01$ & $1.09 \pm 0.08$ & $0.61 \pm 0.06$ \\
\hline$\sigma_{b}$ & $\AA$ & $0.42 \pm 0.02$ & $0.43 \pm 0.01$ & $0.43 \pm 0.08$ & $0.54 \pm 0.06$ \\
\hline$\sigma_{f}$ & $\AA$ & set to be same as $\sigma_{i}$ & $1.16 \pm 0.02$ & set to be same as $\sigma_{i}$ & - \\
\hline$\sigma_{\mathrm{H} 2 \mathrm{O}}$ & $\AA$ & 0.66 & 0.66 & 0.66 & - \\
\hline$d$ & $\AA$ & $2.70 \pm 0.03$ & $2.71 \pm 0.01$ & $2.74 \pm 0.06$ & $2.69 \pm 0.05$ \\
\hline$d_{f}$ & $\AA$ & $2.92 \pm 0.03$ & $3.27 \pm 0.04$ & $2.87 \pm 0.09$ & 2.69 \\
\hline$\rho_{f} / \rho_{e, M e}$ & - & $0.95 \pm 0.01$ & $1.44 \pm 0.02$ & $0.93 \pm 0.05$ & 1 \\
\hline$\rho_{\mathrm{H} 2 \mathrm{O}} / \rho_{e, \mathrm{Me}}$ & - & 0.1 & 0.17 & 0.1 & - \\
\hline$z \mathrm{H} 2 \mathrm{O}$ & $\AA$ & -0.12 & -0.12 & $-0.13 \pm 0.07$ & - \\
\hline
\end{tabular}

a. $E=1.4 \mathrm{~V}$ (this work)

b. $E=1.4 \mathrm{~V}$ (this work)

c. Mean values determined from the values reported in the SI of Temperature-and potential-dependent structure of the mercury-electrolyte interface, Phys. Rev. B, 2016, 93, 165408

$d$. Data reported for In in vacuum at $T=443 \mathrm{~K}$ in Surface structure of liquid metals and the effect of capillary waves: X-ray studies on liquid indium, Phys. Rev. B, 1999, 59, 783 
Table S2. Summary of Voltammetric Features for $\mathrm{GeO}_{2}$ Reduction on $\mathrm{Hg}, \mathrm{Hg}_{1-\mathrm{x}} \mathrm{In}_{\mathrm{x}}$, and In Electrodes

\begin{tabular}{|c|c|c|c|c|c|c|c|c|}
\hline $\begin{array}{l}\text { Electrode } \\
\text { composition }\end{array}$ & $\begin{array}{c}\text { Cathodic peak } \\
\text { potential / V }\end{array}$ & $\begin{array}{c}\text { Cathodic peak } \\
\text { current density / } \\
\mathrm{mA} \mathrm{cm} \\
\end{array}$ & $\begin{array}{c}\text { Integrated cathodic } \\
\text { charge density / } \\
\mathrm{mC} \mathrm{cm}^{-2} \\
\end{array}$ & $\begin{array}{l}\text { Anodic peak } \\
\text { potential / V }\end{array}$ & $\begin{array}{c}\text { Anodic peak } \\
\text { current density / } \\
\mathrm{mA} \mathrm{cm}^{-2} \\
\end{array}$ & $\begin{array}{c}\text { Integrated anodic } \\
\text { charge density } \\
/ \mathrm{mC} \mathrm{cm}^{-2} \\
\end{array}$ & $\begin{array}{c}\text { Faraday Efficiency for } \\
\mathrm{GeO}_{2} \text { Reduction at } E= \\
-1.4 \mathrm{~V} / \%\end{array}$ & $\begin{array}{c}\text { Faraday Efficiency for } \\
\mathrm{GeO}_{2} \text { Reduction at } E= \\
-1.9 \mathrm{~V} / \%\end{array}$ \\
\hline $\mathrm{Hg}$ & -- & 0 & 0 & -- & 0 & 0 & 97.6 & 66.1 \\
\hline $\mathrm{Hg}_{0.7} \operatorname{In}_{0.3}$ & -1.09 & 0.24 & 3.86 & -0.81 & 0.10 & 0.78 & 81.6 & 76.7 \\
\hline $\mathrm{Hg}_{0.5} \operatorname{In}_{0.5}$ & -1.08 & 0.23 & 3.33 & -0.83 & 0.12 & 0.81 & 80.7 & 77.8 \\
\hline $\mathrm{Hg}_{0.3} \operatorname{In}_{0.7}$ & -1.15 & 0.36 & 4.94 & -0.83 & 0.12 & 0.83 & 78.5 & 59.2 \\
\hline In & -1.07 & 0.52 & 7.71 & -0.86 & 0.16 & 1.88 & 56.6 & 4.7 \\
\hline
\end{tabular}

Table S3. Raman Features for As-Prepared Ge from ec-LLS

\begin{tabular}{ccccc}
\hline & \multicolumn{2}{c}{$\mathbf{- 1 . 4} \mathbf{~ V}$} & \multicolumn{2}{c}{$\mathbf{- 1 . 9} \mathbf{~ V}$} \\
\hline $\begin{array}{c}\text { Electrode } \\
\text { compositio }\end{array}$ & $\begin{array}{c}\text { Peak } \\
\text { position }\end{array}$ & fwhm $/ \mathbf{c m}^{-\mathbf{1}}$ & $\begin{array}{c}\text { Peak } \\
\text { position } / \mathbf{c m}^{-}\end{array}$ & $\boldsymbol{f w h m ~} / \mathbf{c m}^{-\mathbf{1}}$ \\
\hline $\mathrm{Hg}$ & 287.4 & 12.3 & 285.9 & 17.6 \\
$\mathrm{Hg}_{0.7} \mathrm{In}_{0.3}$ & 284.5 & 18.1 & 284.7 & 18.4 \\
$\mathrm{Hg}_{0.5} \mathrm{In}_{0.5}$ & 285.4 & 15.4 & 285.1 & 15.9 \\
$\mathrm{Hg}_{0.3} \mathrm{In}_{0.7}$ & 286.9 & 17.9 & 285.4 & 18.9 \\
$\mathrm{In}$ & 289.3 & 12.9 & 288.1 & 19.8 \\
\hline
\end{tabular}


Table S4. Summary of X-ray Diffraction Data for As-Prepared Ge from ec-LLS

\begin{tabular}{|c|c|c|c|c|c|c|c|c|c|c|}
\hline & \multicolumn{5}{|c|}{$-1.4 \mathrm{~V}$} & \multicolumn{5}{|c|}{$-1.9 \mathrm{~V}$} \\
\hline $\begin{array}{l}\text { Electrode } \\
\text { composition }\end{array}$ & $\begin{array}{l}\text { Peak position } \\
\qquad / 2 \theta\end{array}$ & $\begin{array}{l}\text { Grain size } \\
\text { /nm }\end{array}$ & $\begin{array}{c}\text { Average } \\
\text { Grain size } \\
\text { /nm }\end{array}$ & $\begin{array}{c}\text { Peak } \\
\text { intensity ratio }\end{array}$ & Lattice constant / $/ \AA$ & $\begin{array}{l}\text { Peak position } \\
\qquad / 2 \theta\end{array}$ & $\begin{array}{l}\text { Grain size } \\
\text { /nm }\end{array}$ & $\begin{array}{c}\text { Average } \\
\text { Grain size } \\
\text { /nm }\end{array}$ & $\begin{array}{c}\text { Peak } \\
\text { intensity ratio }\end{array}$ & $\begin{array}{l}\text { Lattice constant } \\
\qquad / \AA\end{array}$ \\
\hline \multirow{4}{*}{$\mathrm{Hg}$} & $27.45(0.37)$ & 21.8 & \multirow{4}{*}{$15.2 \pm 4.6$} & 1.00 & \multirow{4}{*}{$5.6628 \pm 0.0041$} & $27.45(0.54)$ & 15.1 & \multirow{4}{*}{$13.1 \pm 1.7$} & 1.00 & \multirow{4}{*}{$5.6562 \pm 0.0039$} \\
\hline & $45.58(0.60)$ & 14.3 & & 1.27 & & $45.58(0.61)$ & 13.9 & & 1.22 & \\
\hline & $53.97(0.66)$ & 13.3 & & 0.87 & & $53.97(0.73)$ & 12.1 & & 0.83 & \\
\hline & $66.34(0.83)$ & 11.4 & & 0.19 & & $66.35(0.85)$ & 11.1 & & 0.23 & \\
\hline \multirow{4}{*}{$\mathrm{Hg}_{0.7} \mathrm{In}_{0.3}$} & $27.37(0.33)$ & 24.4 & \multirow{4}{*}{$18.1 \pm 5.3$} & 1.00 & \multirow{4}{*}{$5.6679 \pm 0.00465$} & $27.37(0.35)$ & 23.1 & \multirow{4}{*}{$17.2 \pm 5.1$} & 1.00 & \multirow{4}{*}{$5.6615 \pm 0.0037$} \\
\hline & $45.42(0.44)$ & 19.2 & & 1.07 & & $45.42(0.46)$ & 18.3 & & 1.16 & \\
\hline & $53.81(0.51)$ & 17.3 & & 0.75 & & $53.81(0.53)$ & 16.8 & & 0.77 & \\
\hline & $66.10(0.82)$ & 11.5 & & 0.21 & & $66.12(0.89)$ & 10.6 & & 0.26 & \\
\hline \multirow{4}{*}{$\mathrm{Hg}_{0.5} \operatorname{In}_{0.5}$} & $27.41(0.41)$ & 19.8 & \multirow{4}{*}{$16.1 \pm 3.0$} & 1.00 & \multirow{4}{*}{$5.6733 \pm 0.00748$} & $27.39(0.47)$ & 17.1 & \multirow{4}{*}{$14.2 \pm 2.5$} & 1.00 & \multirow{4}{*}{$5.6748 \pm 0.0017$} \\
\hline & $45.45(0.51)$ & 16.8 & & 1.54 & & $45.47(0.55)$ & 15.4 & & 1.35 & \\
\hline & $53.83(0.56)$ & 15.7 & & 1.03 & & $53.86(0.67)$ & 13.2 & & 0.95 & \\
\hline & $66.11(0.77)$ & 12.3 & & 0.37 & & $66.18(0.84)$ & 11.2 & & 0.38 & \\
\hline \multirow{4}{*}{$\mathrm{Hg}_{0.3} \operatorname{In}_{0.7}$} & $27.46(0.38)$ & 21.3 & \multirow{4}{*}{$14.8 \pm 4.4$} & 1.00 & \multirow{4}{*}{$5.6791 \pm 0.0061$} & $27.33(0.47)$ & 17.3 & \multirow{4}{*}{$13.5 \pm 2.7$} & 1.00 & \multirow{4}{*}{$5.6865 \pm 0.0039$} \\
\hline & $45.51(0.64)$ & 13.3 & & 1.88 & & $45.37(0.73)$ & 11.7 & & 1.55 & \\
\hline & $53.91(0.72)$ & 12.2 & & 1.46 & & $53.75(0.77)$ & 11.4 & & $`^{1} .03$ & \\
\hline & $66.23(0.77)$ & 12.3 & & 0.48 & & $66.06(0.70)$ & 13.5 & & 0.26 & \\
\hline \multirow{4}{*}{ In } & $27.21(0.49)$ & 16.6 & \multirow{4}{*}{$9.7 \pm 5.0$} & 1.00 & \multirow{4}{*}{$5.6806 \pm 0.0028$} & $27.19(0.53)$ & 15.2 & \multirow{4}{*}{$10.1 \pm 3.5$} & 1.00 & \multirow{4}{*}{$5.6716 \pm 0.0006$} \\
\hline & $45.22(0.85)$ & 10.1 & & 0.87 & & $45.17(0.85)$ & 10.1 & & 0.75 & \\
\hline & $54.24(1.73)$ & 5.1 & & 0.70 & & $54.28(1.17)$ & 7.6 & & 0.73 & \\
\hline & 66.95 (1.37) & 6.9 & & 0.46 & & $66.92(1.21)$ & 7.8 & & 0.71 & \\
\hline
\end{tabular}

